\title{
Correction to: Majorization Results for Subclasses of Starlike Functions Based on the Sine and Cosine Functions
}

\author{
Huo Tang ${ }^{1} \cdot$ H. M. Srivastava ${ }^{2,3} \cdot$ Shu-Hai Li ${ }^{1} \cdot$ Guan-Tie Deng ${ }^{4}$ \\ Published online: 5 October 2019 \\ () Iranian Mathematical Society 2019
}

\section{Correction to: Bulletin of the Iranian Mathematical Society https://doi.org/10.1007/s41980-019-00262-y}

Very recently, Tang et al. (original article) have studied two majorization results for the subclasses $S_{s}^{*}$ and $S_{c}^{*}$ of starlike functions, which are, respectively, connected with the sine and cosine functions. Now, we correct the definition of the class $S_{c}^{*}$ and the corresponding majorization result (Theorem 2.2 in the original article).

\section{Definition and main result}

In the original version of this article, the authors introduced the following class $S_{c}^{*}$ :

The original article can be found online at https://doi.org/10.1007/s41980-019-00262-y.

Huo Tang

thth2009@163.com

H. M. Srivastava

harimsri@math.uvic.ca

Shu-Hai Li

lishms66@163.com

Guan-Tie Deng

denggt@bnu.edu.cn

1 School of Mathematics and Statistics, Chifeng University, Chifeng 024000, Inner Mongolia, People's Republic of China

2 Department of Mathematics and Statistics, University of Victoria, Victoria, BC V8W 3R4, Canada

3 Department of Medical Research, China Medical University Hospital, China Medical University, Taichung 40402, Taiwan, Republic of China

4 School of Mathematical Sciences, Beijing Normal University, Beijing 100875, People's Republic of China 


$$
S_{c}^{*}:=\left\{f: f \in \mathcal{A} \text { and } \frac{z f^{\prime}(z)}{f(z)}<1+\cos z\right\} .
$$

and obtained the corresponding majorization result (Theorem 2.2 in the original article).

Theorem 2.2 Let the function $f \in \mathcal{A}$ and suppose that $g \in S_{c}^{*}$. If $f(z) \ll g(z)$, then, for $|z| \leqq r_{2}$,

$$
\left|f^{\prime}(z)\right| \leqq\left|g^{\prime}(z)\right|,
$$

where $r_{2}$ is the smallest positive root of the following equation:

$$
\left(1-r^{2}\right)(1-\cosh r)-2 r=0 .
$$

Unfortunately, the authors found some minor mistakes (the definition of the class $S_{c}^{*}$ and Theorem 2 in the original article) and correct them as follows.

1. The definition of the class $S_{c}^{*}$ is corrected as

$$
S_{c}^{*}:=\left\{f: f \in \mathcal{A} \quad \text { and } \quad \frac{z f^{\prime}(z)}{f(z)} \prec \cos z\right\} .
$$

2. Base on the above definition (1.5'), we have rewritten (Theorem 2.2 in the original article) as Theorem $2.2^{\prime}$ and give the proof.

Theorem 2.2' Let the function $f \in \mathcal{A}$ and suppose that $g \in S_{c}^{*}$. If $f(z) \ll g(z)$, then, for $|z| \leqq r_{2}^{\prime}$,

$$
\left|f^{\prime}(z)\right| \leqq\left|g^{\prime}(z)\right|
$$

where $r_{2}^{\prime}$ is the smallest positive root of the following equation:

$$
\left(1-r^{2}\right) \cos r-2 r=0 .
$$

Proof Since $g \in S_{c}^{*}$, from $\left(1.5^{\prime}\right)$ and the subordination relationship, we have

$$
\frac{z g^{\prime}(z)}{g(z)}=\cos (\omega(z))
$$

where $\omega(z)$ is defined as (2.2) of Theorem 1 in the original article.

Similar to (2.3) (see Theorem 1 in the original article), we easily verify that

$$
\cos r \leqq \cos R \leqq|\cos (\omega(z))|=\left|\cos \left(\operatorname{Re}^{i t}\right)\right| \leqq \cosh R \leqq \cosh r,
$$

where $\omega(z)=\operatorname{Re}^{i t}$ with $R \leqq|z|=r<1$ and $-\pi \leqq t \leqq \pi$. 
Combining $\left(2.9^{\prime}\right)$ and $\left(2.10^{\prime}\right)$, it is easy to get that

$$
\left|\frac{g(z)}{g^{\prime}(z)}\right| \leqq \frac{r}{\cos r}
$$

Applying (2.6) (see Theorem 1 in the original article) as well as (2.11') in (2.5), (see Theorem 1 in the original article), we immediately show that

$$
\left|f^{\prime}(z)\right| \leqq\left[|\varphi(z)|+\frac{1-|\varphi(z)|^{2}}{1-r^{2}} \cdot \frac{r}{\cos r}\right]\left|g^{\prime}(z)\right| .
$$

Next, according to (2.7) (see Theorem 1 in the original article) and just as the proof of Theorem 1 in the orignal article, we can deduce the required result (2.8'). Hence we have completed the proof of Theorem 2.2'.

Remark In the original version of this paper, replacing $\varphi(z)=1+\cos z$ by $\varphi(z)=\cos$ $z$ in the introduction (Page 4 in the original article), and the concluding remarks (Page 7 in the original article).

Acknowledgements We would like to thank Prof. Zhi-Gang Wang and Dr. Rahim Kargar for their valuable comments and corrections.

Publisher's Note Springer Nature remains neutral with regard to jurisdictional claims in published maps and institutional affiliations. 